\title{
EFICACIA DEL FENTANILO EN ANESTESIA RAQUÍDEA PARA EL DOLOR AGUDO POSTOPERATORIO.
}

\author{
EFFICACY OF FENTANYL IN SPINAL ANESTHESIA FOR ACUTE \\ POSTOPERATIVE PAIN.
}

\author{
Cárdenas-Delgado Pool ${ }^{1, a}$ \\ 1. Médico cirujano. \\ a. Hospital Regional de Ayacucho, Huamanga, Perú.
}

\section{Correspondencia:}

Cárdenas-Delgado Pool

Dirección:

Asociación Quijano Mendivil D -6, Huamanga - Ayacucho

Número de celular: 9741088755

Correo Electrónico:

poolcardel@hotmail.com

Contribuciones De Autoría:

CDP: Contribuciones en la concepción y diseño del manuscrito, recolección, análisis e interpretación de los datos, redacción y revisión crítica del contenido del manuscrito y aprobación final del artículo.

Conflicto De Intereses: No declarados.

Financiamiento: Autofinanciado.

\section{Como Citar}

Cárdenas Delgado Pool. Eficacia del fentanilo en anestesia raquídea para el dolor agudo postoperatorio. Rev méd panacea. 2019; 8(3): 116-120. DOI: https://doi.org/10.35563/rmp.v8i3.195

Recibido: 17 - 08 - 2019

Aceptado: 20 - 09 - 2019

Publicado: 22 - 09 - 2019

\section{RESUMEN}

Objetivo: El dolor agudo postoperatorio es un conjunto de percepciones sensoriales, emocionales y mentales desagradables, asociadas a respuestas autonómicas, psicológicas y conductuales, precipitadas por el acto quirúrgico. Objetivo: Generar conocimiento sobre la eficacia del fentanilo en anestesia raquídea para el dolor agudo postoperatorio. Material y métodos: Es un estudio descriptivo de búsqueda bibliografía y se ha realizado en Pubmed, Medline, Scielo, bibliotecas de universidades nacionales e internacionales. Resultados: La asociación de fentanilo a los anestésicos locales para la anestesia subaracnoidea, reduciendo la dosificación de anestésico local y manteniendo el tiempo de su recuperación y así de esta manera mejorar la calidad, la tasa de éxito de pequeñas dosis de los mismos, con ello, la incidencia de complicaciones hemodinámicas. La administración subaracnoidea de anestésicos locales y opioides, provocan eventos adversos, prurito (30 \%), náuseas y vómito (25\%), retención urinaria (10-53\%), depresión respiratoria (3\%). Las diferentes dosis de fentanilo asociado a bupivacaína $0.5 \%$, en la raquianestesia disminuyen la intensidad de dolor según EVA, en el trans-operatorio y el manejo del dolor agudo post-operatorio en los diferentes actos quirúrgicos. Conclusión: Se concluye que la asociación de bupivacaína 0,5\% más fentanilo, mediante la técnica raquídea, en pacientes intervenidos quirúrgicamente, resulta eficaz, debido a que permite obtener un menor tiempo de latencia para el bloqueo con una duración prolongada, manteniendo una adecuada hemodinamia y estabilidad respiratoria, y también reduciendo el dolor postoperatorio y sus posibles reacciones adversas.

Palabras clave: Fentanilo, anestesia raquídea, bupivacaina, dolo agudo postoperatorio.

\section{ABSTRACT}

Introduction: Acute postoperative pain is a set of unpleasant sensory, emotional and mental perceptions associated with autonomic, psychological and behavioral responses precipitated by the surgical act. Objective: Generate knowledge about the efficacy of fentanyl in spinal anesthesia for acute postoperative pain. Material and methods: It is a descriptive study of literature search and has been carried out in Pubmed, Medline, Scielo, libraries of national and international universities. Results: The association of fentanyl with local anesthetics for subarachnoid anesthesia, reduces the dose of local anesthetic, without prolonging the recovery time, improving the quality, the success rate of small doses of them, with this, the incidence of hemodynamic complications. The subarachnoid administration of local anesthetics and opioids, cause adverse events, itching (30\%), nausea and vomiting (25\%), urinary retention (10-53\%), respiratory depression (3\%), all this as a result of interactions with opioid receptors at the brain level. The different doses of fentanyl associated with $0.5 \%$ bupivacaine, in spinal anesthesia reduce the intensity of pain according to EVA, in the trans-operative period and the management of acute post-operative pain in the different surgical acts. Conclusion: It is concluded that the association of $0.5 \%$ bupivacaine plus fentanyl, through the spinal technique, in surgically treated patients, is effective, since it allows to reach a shorter time of onset of the blockade and longer duration of the block, maintaining adequate hemodynamic and respiratory stability, the time it reduces acute post-operative pain and adverse reactions.

Keywords: Fentanyl, spinal anesthesia, bupivacaine, acute postoperative pain. 


\section{INTRODUCCIÓN}

El dolor agudo postoperatorio es un conjunto de percepciones sensoriales, emocionales y mentales desagradables, asociadas a respuestas autonómicas, psicológicas y conductuales que han sido precipitadas como consecuencia del acto quirúrgico (1).

Se sabe que muchos factores influyen en la experiencia del dolor, como el sexo, la edad, la cultura y las experiencias anteriores. El significado del dolor es algo que la persona describe, para ello controlar el dolor requiere una buena cooperación entre el paciente y el médico y el personal clínico (2).

El dolor intenso después de la cirugía sigue siendo un problema importante, y ocurre en el $20-40 \%$ de los pacientes. A pesar de los numerosos estudios publicados, se desconoce el grado de dolor después de muchos tipos de cirugía en la práctica clínica diaria. Para mejorar la terapia del dolor post-operatorio y desarrollar protocolos de tratamiento del dolor optimizado para cada procedimiento, primero se deben identificar los tipos de cirugía (3).

El tratamiento del dolor post-operatorio continúa siendo una importante preocupación para los profesionales sanitarios, así como para las instituciones sanitarias. Desgraciadamente, a pesar de contar con diversas estructuras organizativas y una gran variedad de recursos terapéuticos, no se ha conseguido disminuir significativamente la prevalencia del dolor agudo posoperatorio. Un correcto control del dolor posoperatorio es un indicador de calidad de los hospitales (4).

La cantidad de dolor que sufre un paciente después de la cirugía está relacionada con la extensión del daño tisular y el sitio de la cirugía. El buen control del dolor es importante para prevenir resultados negativos como hipertensión, isquemia miocárdica, arritmias, insuficiencia respiratoria, íleo y mala cicatrización de la herida (5).

Un inadecuado control del dolor post-operatorio puede ensombrecer el posible éxito del tratamiento quirúrgico, retrasa la recuperación y prolonga la estancia hospitalaria, retrasando la reincorporación de nuestros pacientes a su vida familiar y laboral. Por otra parte, no debemos olvidar la repercusión económica que esto ocasiona. Y Además mencionar el incremento de la posibilidad de cronicidad del dolor por parte del dolor post operatorio mal controlado (6).

Aunque, en la actualidad, la anestesia espinal tiene un lugar muy importante en el manejo anestésico regional de los pacientes quirúrgicos debido a las propiedades únicas de la técnica. Estas propiedades incluyen la rapidez y el control de inicio, así como la duración de la anestesia, efectos secundarios y complicaciones mínima facilidad de administración, y un bajo costo (7).

La adición de opiáceos a las soluciones anestésicas espinales persigue mejorar la calidad de la anestesia intra-operatoria y prolongar la analgesia post-operatoria; el fentanilo y la morfina, respectivamente, son los opiáceos más utilizados para estos propósitos. El fentanilo, a las dosis actualmente recomendadas, menores de 20ug, mejora la calidad de la anestesia y disminuye la dosis de anestésico local (8).

Existen muchos estudios donde mencionan el uso de opioides conjuntamente con los anestésicos locales para realizar bloqueos regionales, proporcionando una excelente analgesia post-operatoria. Dentro de las cuales la combinación de bupivacaina con fentanilo termina siendo una de las más aceptadas debido a su efectividad anestésica y analgésica durante la anestesia raquídea. La hipotensión arterial es la complicación más frecuente secundaria a la anestesia espinal conjuntamente de bradicardia, prurito facial, escalofríos (temblores), sensación nauseosa y vómitos, que son relacionados por el uso del fentanilo (9).

El objetivo principal de esta revisión bibliográfica es generar conocimiento sobre la eficacia del fentanilo usado en la anestesia raquídea, para el manejo del dolor agudo post-operatorio.

\section{MATERIAL Y MÉTODOS.}

La búsqueda bibliográfica se ha realizado en Pubmed, Medline, Scielo, bibliotecas de universidades nacionales e internacionales. Como criterios de selección se tomó en cuenta información publicada en dichas fuentes dada la confiabilidad de las mismas, con una antigüedad no mayor de 5 años.

\section{RESULTADOS.}

Orsolini JL. Y Col. (Boliovia 2016). Anestesia regional raquidea con bupivacaína combinada con fentanilo y morfina en cirugía abdominal baja. Se realizó un estudio descriptivo prospectivo con 100 pacientes de cirugía ginecológica, urológica y general que cumplían los criterios de inclusión en el Hospital Obrero $\mathrm{N}^{\circ} 2$, de Julio a diciembre del 2015. Con el esquema combinado que se ha utilizado, se obtiene una analgesia posoperatoria satisfactoria hasta las 12 horas donde los valores de EVA se encuentran dentro de parámetros aceptables (EVA 3) antes del inicio de la analgesia de rescate. Se utilizó analgesia de rescate en el $13 \%(13 / 100)$ de los pacientes que se administró por vía endovenosa entre las 6 a 12 hrs post-operatorias con ketorolaco 60mg Los efectos adversos valorados fueron prurito, náuseas, vómitos, retención urinaria y depresión respiratoria. Mayor frecuencia de nauseas (19\%) y prurito (14\%). Concluimos que la anestesia raquídea combinada con bupivacaína pesada al $0.5 \%$ más fentanilo y morfina en cirugía abdominal baja otorga mayores beneficios en relación a la anestesia raquídea convencional (10).

Bofill P. y Col. (Cuba 2017). Anestesia subaracnoidea con dosis variables de bupivacaína con fentanilo, en la resección trans-uretral de la próstata. Se realizó un estudio descriptivo cuantitativo, longitudinal, prospectivo en 200 pacientes programados para dicha cirugía e indicación de anestesia subaracnoidea con bupivacaína, los cuales fueron distribuidos de forma 
aleatoria en cuatro grupos. Tanto el bloqueo sensitivo como motor fue más rápido en los pacientes de los Grupos B $(3,0 ; 11,2)$ y $(7,1 ; 8,3)$, Grupo C $(5,0 ; 9,4)$ y $(6,2$; $9,4)$ y Grupo $D(6,1 ; 8,3)$ y $(4,7 ; 10,9)$; mientras que el Grupo A fue de $(6,0 ; 8,5)$ y $(9,9 ; 11,7)$, respectivamente. Se observó que en la totalidad de los pacientes de los Grupos C y D se obtuvo anestesia de buena calidad. La administración intratecal de 7,5 mg de bupivacaína con $25 \mu \mathrm{g}$ de fentanilo produjo anestesia satisfactoria, disminuyó el periodo de latencia, mantuvo mejor estabilidad hemodinámica, prolongó la analgesia post-operatoria y se asoció a menor incidencia de complicaciones peri operatorias (11).

Linares S. y Col. (El Salvador 2018). Caracterización y manejo de los episodios de dolor postquirúrgico en Cirugía y Ortopedia. Estudio analítico de corte transversal en pacientes postquirúrgicos de ortopedia y cirugía del Hospital Nacional San Rafael de El Salvador, durante febrero-abril 2013; evaluando sistemáticamente la intensidad del dolor a las 2 h, 6 h, 12 h, y 24 h; utilizando la Escala Visual Análoga y Verbal Numérica. El analgésico prescrito no fue pertinente en la mayoría de los casos generando persistencia del dolor no controlado después de las 24 horas postquirúrgicas (12).

Piñeiro E. y Col. (Cuba 2017). Combinación de bupivacaína hiperbárica y fentanilo como anestesia espinal en pacientes con fractura de cadera. Se realizó un estudio observacional, descriptivo y comparativo de 54 pacientes con fractura de cadera, atendidos en el Servicio de Anestesiología del Hospital Provincial Docente Clínico quirúrgico "Saturnino Lora Torres" de Santiago de Cuba, desde enero de 2011 hasta diciembre de 2013. La muestra fue dividida en 2 grupos: Los del A recibieron bupivacaína hiperbárica a 0,4\% más fentanilo; los del B, bupivacaína hiperbárica a 0,4\% solamente. Se observó que el tiempo de inicio y duración del bloqueo fue mejor en los integrantes del grupo A; asimismo, las variaciones hemodinámicas y respiratorias, así como los efectos adversos no mostraron diferencias entre los grupos. La analgesia post-operatoria fue superior en el primer grupo, de manera que esta técnica resultó efectiva. Se concluye que la asociación de bupivacaína hiperbárica a 0,4\% más fentanilo, mediante la técnica espinal, en pacientes operados por fractura de cadera, resulta efectiva, pues permite alcanzar menor tiempo de inicio del bloqueo y mayor duración de este, mantiene adecuada estabilidad hemodinámica y respiratoria, a la vez que reduce el dolor post-operatorio y las reacciones adversas (13).

Sarmiento KA. (Ecuador 2014). Anestesia raquídea con dosis mínimas efectivas de bupivacaína hiperbárica al $0.5 \%$ más opioide versus anestesia raquídea con dosis habituales a pacientes sometidos a artroscopias del Hospital Manuel Ignacio Monteros de Loja, abril 2013 enero 2014. Estudio clínico, prospectivo simple ciego de 60 pacientes ASA I y II distribuidos en dos grupos. El grupo control recibió 10 mg de bupivacaína 0,5\% hiperbárica, el grupo de dosis baja se le administraron 5 mg de bupivacaína 0,5\% hiperbárica ambos con 25ug de fentanilo. El empleo de dosis bajas de bupivacaína hiperbárica al $0.5 \%$ con adición del opioide es una buena alternativa que permite obtener una analgesia quirúrgica satisfactoria, con recuperación motora rápida, un buen nivel metamérico y con buena estabilidad hemodinámica (14).

Ulco SF. (Perú 2018). Eficacia de bupivacaína hipobárica a dosis de $1.5 \mathrm{mg}$ combinada con fentanilo en raquianestesia para legrados uterinos. Ensayo clínico realizado en el Centro Quirúrgico del Hospital Provincial Docente Belén de Lambayeque entre junio del 2017 a marzo del 2018 en 60 pacientes con diagnóstico de aborto incompleto espontaneo a quienes se les realizó legrado uterino con raquianestesia con dosis ultrabajas de bupivacaína hipobárica 0.5 \% más 10 ug de fentanilo, las cuales fueron divididas aleatoriamente en 2 grupos de 30 pacientes cada uno: el grupo control recibió 2 mg de bupivacaína y el experimental $1.5 \mathrm{mg}$. No hubo cambios relevantes clínicamente de la hemodinamia y SO2 en los grupos de estudio, ningún paciente hizo bradicardia e hipotensión. Las pacientes ingresaron con un EVA promedio de 3.63 a 4.13 en ambos grupos y después de la raquianestesia el dolor llegó a un EVA=0.06 ( $p=0.000)$, el máximo nivel de bloqueo sensitivo alcanzado en el grupo experimental fue de T8 -T6 y en el control de T6-T4, no hubo bloqueo motor (Bromage $=0$ ) ni efectos colaterales en ninguna paciente. El grado de Confort en ambos grupos fue bueno. La dosis de $1.5 \mathrm{mg}$ de bupivacaína $0.5 \%$ hipobárica SI es eficaz como la de $2 \mathrm{mg}$, ambas combinadas con fentanilo en la raquianestesia para la realización de Legrados uterinos (15).

Estrada D. (Cuba 2017). Fentanilo y bupivacaína al 0,5\% en ancianos con fractura de cadera operados con anestesia subaracnoidea. Se realizó un estudio longitudinal, prospectivo, en el Hospital Ernesto Guevara de la Serna, entre enero-diciembre de 2015. La muestra por 47 pacientes con estado físico ASA II y III, operados utilizando anestesia subaracnoidea con dosis de bupivacaína 0,5\% 8 mg más fentanilo 25 mcg.. La totalidad de los casos refirieron prurito facial leve como efecto adverso (16).

\section{DISCUSIÓN.}

La asociación de opioides como el fentanilo a los anestésicos locales para la anestesia subaracnoidea constituye una práctica encaminada a reducir la dosis de anestésico local, sin prolongar el tiempo de recuperación y mejorar la calidad y la tasa de éxito de pequeñas dosis de los mismos $y$, con ello, la incidencia de complicaciones hemodinámicas, como la hipotensión arterial (16).

A pesar de que la administración subaracnoidea de anestésicos locales y opioides, es una excelente técnica 
anestésica y de control del dolor, se describen eventos adversos, entre ellos los más comunes son prurito (30\%), náuseas y vómito (25\%), retención urinaria (10-53\%) y el más temido, la depresión respiratoria (3\%), todo esto como resultado de interacciones con los receptores opioides a nivel cerebral $(11,13)$.

Las diferentes dosis de fentanilo asociado a bupivacaína $0.5 \%$, en la raquianestesia si disminuyen significativamente la intensidad de dolor según EVA (Escala Visual Análoga) en el trans-operatorio y el correspondiente manejo del dolor agudo post-operatorio en los diferentes actos quirúrgicos (15).

\section{CONCLUSIÓN}

Se concluye que la asociación de bupivacaína 0,5\% más fentanilo, mediante la técnica raquídea, en pacientes intervenidos quirúrgicamente, resulta eficaz, debido a que permite obtener un menor tiempo de latencia para el bloqueo con una duración prolongada, manteniendo una adecuada hemodinamia y estabilidad respiratoria, y también reduciendo el dolor postoperatorio y sus posibles reacciones adversas.

El fentanilo debido a su liposolubilidad, el cual le permite llegar al líquido cefaloraquideo en forma rápida y así de esta manera lograr acortar el tiempo de latencia y prolongar el efecto analgésico hasta un máximo de 6 horas. Motivo por el cual es considerado como uno de los opioides más usados para obtener muy buena analgesia y anestesia después de su administración intratecal. 


\section{REFERENCIAS BIBLIOGRÁFICAS}

1. Aguilar JL, Montes A, Benito C, Caba F y Margarit C. Manejo farmacológico del dolor agudo post-operatorio en España. Datos de la encuesta nacional de la Sociedad Española del Dolor (SED). Rev. Soc. Esp. Dolor. España 2018; 25(2): 70-85. https://www.resed.es/Documentos/ArticulosNew/04_ OR_Aguilar.pdf

2. Hussain Z, Karvandian K, Maghsoudloo M, Muhy $H$. The Role of Opioids and Non-Opioids in Postoperative Pain Relief: A Narrative Review. Archives of Anesthesiology and Critical Care. Irán 2018; 4(1): 430-435.

http://aacc.tums.ac.ir/index.php/aacc/article/view/13 8

3. Gerbershagen HJ, Aduckathil S, Van Wijck AJ, Peelen LM, Kalkman CJ, Meissner W. Pain intensity on the first day after surgery: a prospective cohort study comparing 179 surgical procedures. Anesthesiology. Netherlands 2013; 118(4): 934-944.

https://www.ncbi.nlm.nih.gov/pubmed/23392233

4. García JL, Neira RF. El tratamiento del dolor posoperatorio ¿Un problema sin resolver?. Revista de la Sociedad Española del Dolor. España 2018; 25(2): 63-65.

http://scielo.isciii.es/scielo.php?script=sci_arttext\&pid =S1134-80462018000200063

5. Corke P. Postoperative pain management. Australian Prescriber. Australia 2013; 36(6): 202-205.

https://www.nps.org.au/assets/4811a27845042173-0 0a4ff09097b-postoperative-pain-management_36-20 2.pdf

6. Montes A, Roca G, Sabate S. Genetic and clinical factors associated with chronic postsurgical pain after hernia repair, hysterectomy, and thoracotomy: a two-year multicenter cohort study. Anesthesiology. USA 2015; 122(5): 1123-1141.

https://anesthesiology.pubs.asahq.org/article.aspx?ar ticleid $=2135169$

7. Jiménez KP. Comparación del uso espinal de levobupivacaína vs levobupivacaína más fentanilo, en artroscopias de rodilla ambulatorias. Revista Médica de la Universidad de Costa Rica. Costa Rica 2017; 11(2): 25-33.

https://revistas.ucr.ac.cr/index.php/medica/article/vie w/34576/34109

8. Herrera PJ, Garzón JF. Opiáceos intratecales y depresión respiratoria: ¿Un mito en obstetricia?. Revista Colombiana de Anestesiología. Colombia 2015; 43(1): 101-103.

https://www.sciencedirect.com/science/article/pii/S01 2033471400135X

9. Lachicott $Y$, López $N$, Donis $M$, Companioni D, Ortiz N, Ferriol PC. Eficacia clínica de la bupivacaína al 0,5\% asociada a dosis de fentanilo en la anestesia espinal para cesárea. Mediciego; Cuba 2017; 23(4): 20-28. http://www.revmediciego.sld.cu/index.php/medicieg o/article/view/693/1166
10. Orsolini JL, Ardaya M. Anestesia regional raquidea con bupivacaína combinada con fentanilo y morfina en cirugía abdominal baja. Revista Médica. Bolivia 2016; 23(1): 24-29.

http://www.revistasbolivianas.org.bo/pdf/rmcba/v2 3n1/v23n1_a06.pdf

11. Bofill $P$, Valenzuela $K$, Carballea $Y$, Ramos $Y$, Bofill J. Anestesia subaracnoidea con dosis variables de bupivacaína con fentanilo, en la resección transuretral de la próstata. Revista Médica Electrónica. Cuba 2017; 39(3): 495-506. http://scielo.sld.cu/pdf/rme/v39n3/rme080317.pdf

12. Linares $S$, Lahud $S$, García Z. Caracterización y manejo de los episodios de dolor post-quirúrgico en Cirugía y Ortopedia. SINAPSIS UJMD. El Salvador 2018; 8(1): 4-11.

http://www.sinapsisujmd.org/index.php/sinujmd/art icle/view/58/7

13. Piñeiro $E$, Domínguez $E J$, Muguercia $K$, Fabré $U$. Combinación de bupivacaína hiperbárica y fentanilo como anestesia espinal en pacientes con fractura de cadera. MEDISAN. Cuba 2017; 21(3): 250-257. http://scielo.sld.cu/pdf/san/v21n3/san02203.pdf

14. Sarmiento KA. Anestesia raquídea con dosis mínimas efectivas de bupivacaína hiperbárica al 0.5\% más opioide versus anestesia raquídea con dosis habituales a pacientes sometidos a artroscopias del Hospital Manuel Ignacio Monteros de Loja, abril 2013 - enero 2014 [Tesis Post-Grado]. Ecuador: Universidad Nacional de Loja. Carrera de Medicina Humana; 2014.

https://dspace.unl.edu.ec/jspui/bitstream/12345678 9/18751/1/Dra.\%20Karla\%20Alexandra\%20Sarmient o\%20\%C3\%81lvarez.pdf

15. Ulco SF. Eficacia de bupivacaína hipobárica a dosis de $1.5 \mathrm{mg}$ combinada con fentanilo en raquianestesia para legrados uterinos (Tesis Post-Grado). Perú: Universidad Nacional Pedro Ruiz Gallo. Facultad de Medicina Humana; 2018. http://repositorio.unprg.edu.pe/bitstream/handle/U NPRG/2967/BC-TES-TMP-1786.pdf? sequence $=1$ \&is Allowed $=y$

16. Estrada D, Barreda JL, Gómez I, Cruz D, Rojas S. Fentanilo y bupivacaína al $0,5 \%$ en ancianos con fractura de cadera operados con anestesia subaracnoidea. Revista Electrónica Dr. Zoilo E. Marinello Vidaurreta. Cuba 2017; 42(3): 1-6. http://revzoilomarinello.sld.cu/index.php/zmv/articl e/view/1100/pdf_403

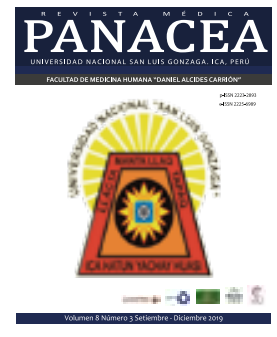

